\title{
Pourquoi les décisions de limitation des traitements en réanimation ne sont elles pas partagées avec les professionnels assurant les soins de l'enfant avant et après la réanimation?
}

Titre court : Pourquoi les décisions de LAT prises en réanimation ne sont elles pas pérennes ?

Robin Cremer ${ }^{1,2}$, Alexandra Binoche ${ }^{1}$, Grégoire Moutel ${ }^{2}$, Béatrice Fourestié ${ }^{1}$, A Botte ${ }^{1}$, Catherine Le Grand-Sébille ${ }^{3}$, Francis Leclerc ${ }^{1}$

1 : Réanimation pédiatrique, Hôpital Jeanne de Flandre, CHU de Lille, 59037 Lille France.

2 : Réseau de recherche en éthique de l'INSERM, Laboratoire d'éthique médicale, faculté de médecine, 45 rue des Saints-Pères, 75007 Paris, France.

3 : Faculté de médecine de Lille, 59045, Lille cedex, France.

Correspondance: $\quad$ Robin Cremer

Réanimation pédiatrique. Hôpital Jeanne de Flandre

CHRU Lille - 59037 Lille cedex, France

Tél. 0320446879 - Télécopie : 0320444719

Mél. $\underline{\text { r-cremer@chru-lille.fr }}$ 


\section{Résumé}

Une précédente étude avait montré que les décisions de limitation ou d'arrêt des traitements (LAT) prises en réanimation n'étaient pas partagées par les services destinataires des enfants survivants. Objectifs de l'étude : mettre à jour les freins à cette prise en compte et les analyser dans une perspective d'amélioration de la continuité des soins. Méthode : étude qualitative réalisée à partir d'entretiens ouverts auprès de médecins seniors extérieurs à la réanimation confrontés au protocole de décisions de LAT. Résultats : neuf médecins ont été interrogés (neuropédiatres, neurochirurgiens pédiatriques et onco-hématologues pédiatres). Le corpus d'entretien représentait 80 pages dactylographiées. Trois situations génératrices de tensions ont été identifiées. La plus fréquemment rapportée était leur intervention à titre d'expert chargé d'établir un pronostic à la demande des réanimateurs. Les deux autres correspondaient à l'admission de leurs patients en réanimation lorsqu'ils devaient justifier de l'existence d'un projet de soin et à la sortie lorsque le patient survivait après une décision de LAT. Conclusion : la modélisation du raisonnement médical utilisée pour la formalisation des LAT en réanimation est source de tensions quand elle est confrontée aux modélisations des autres spécialités. Ces modélisations représentent des modes différents d'intégration de la part de subjectivité de tout raisonnement médical. La réintégration de cette subjectivité dans la pratique, favoriserait la délibération autour du malade qui est nécessaire à la continuité de sa prise en charge.

Mots clé : limitation ou arrêt de traitements, handicap, réanimation pédiatrique, éthique 


\begin{abstract}
:
The fact that some children may survive despite a decision of limitation of treatments (DLT) was made in the Paediatric Intensive Care Unit (PICU) is a recent concern. A previous study pointed out that the teams in charge of children after the PICU stay did not take previous PICU DLT into account. Purpose: to identify what are the difficulties for different units to cooperate with the PICU team, in order to increase continuity of care for these children. Study design: qualitative single centre study, using semistructured interviews with physicians in charge of children who were discharged from the PICU despite a DLT was made. Results: interviews concerned nine paediatric subspecialists (hemato-oncologists, neurologists and neurosurgeons) and produced about 80 pages of typed text. Three straining situations were identified by these physicians. The most frequently reported situation was to be asked by the PICU team to give an expert advice on prognosis, before a DLT was made. The two others were to have to justify a true care plan for children who were transferred to the PICU, and to conceive a new care plan for children who were discharged from the PICU after a DLT was made. Conclusion: the medical reasoning model that is used for DLTs in PICU generates straining situations when confronted to the models that are used by other specialties. These models are representing various expressions of subjectivity, which is associated with any medical decision. Acknowledging this fact could facilitate its integration in clinical practice and should improve authentic debates that are necessary to ensure continuity of care for these children.
\end{abstract}


Dans les pays développés 75 à 80 \% des enfants meurent à l'hôpital, essentiellement en réanimation $[11,12,28]$, où 30 à $40 \%$ des décès surviennent à la suite d'une décision médicale de limitation ou d'arrêt des traitements (LAT) $[11,12,28]$.

Les pédiatres réanimateurs, regroupés au sein du Groupe Francophone de Réanimation et Urgence Pédiatrique (GFRUP), membre de la Société Française de Pédiatrie (SFP) et la Société de Réanimation de Langue Française (SRLF), ont mené une réflexion sur ce sujet de 1998 à 2000. Dix groupes de travail, constitués de professionnels francophones de la réanimation pédiatrique (médecins, infirmières et cadres infirmiers), de représentants de familles ayant perdu un enfant en réanimation, de professionnels des soins palliatifs et de personnes conduisant une recherche dans le domaine de l'éthique médicale ont réfléchi sur cette problématique. Le produit de cette réflexion a été édité par la Fondation de France en avril 2002, sous la forme d'un fascicule intitulé : «Limitation ou arrêt des traitements en réanimation pédiatrique. Repères pour la pratique », qui a été diffusé à toutes les unités de réanimation pédiatrique francophones [7]. Cette démarche a été poursuivie par la rédaction de recommandations, validées par la commission d'éthique de la SFP. Celles-ci ont été largement diffusées, et publiées dans les Archives de Pédiatrie en octobre 2005 [8] ; elles sont consultables sur le site Internet du GFRUP à l'adresse suivante : http://www.gfrup.com/fin vie/gfrup fin vie.htm.

Durant l'année 2005, la législation française a évolué et a donné un cadre légal à ces recommandations [9], en rendant possible la limitation et l'arrêt de soins jugés futiles et inutiles, protégeant ainsi les médecins réanimateurs contre d'éventuelles poursuites par le parquet. Le code de santé publique, modifié par cette loi et par ses décrets d'application [10], précise que le médecin en charge du patient doit pour cela : $1^{\circ}$ ) prendre une décision collégiale, c'est-à-dire en concertation avec l'équipe soignante et avec l'avis motivé d'un médecin consultant n'ayant aucun lien de nature hiérarchique avec lui, $2^{\circ}$ ) recueillir l'avis des titulaires de l'autorité parentale, et $3^{\circ}$ ) notifier dans le dossier de l'enfant les avis recueillis, la nature et le sens des concertations qui ont lieu au sein de l'équipe ainsi que les motifs de la décision. 
De 2002 à 2005, le service de réanimation pédiatrique de Lille a mené une étude prospective d'autoévaluation des pratiques, pour évaluer l'applicabilité pratique des recommandations du GFRUP [11]. La population concernée était l'ensemble des malades pour lesquels une réflexion éthique pendant le séjour était jugée nécessaire par le médecin senior responsable des soins et l'inclusion se faisait dès l'origine du questionnement. La moitié de ces réflexions donnaient lieu à une décision formalisée ; pour l'autre moitié, les causes de l'interruption du processus se répartissaient en deux groupes d'effectif égal : soit un décès par échec de réanimation, soit une « amélioration clinique » suivie d'un transfert dans un autre service. Par ailleurs, lorsque le processus donnait lieu à une décision de ne pas réanimer en cas d'arrêt cardiaque, ou de limitation des traitements actifs, la survie était observée dans $35 \%$ des cas. La mise en évidence d'une population ayant survécu après une réflexion sur la pertinence des traitements de réanimation, voire après une décision de LAT posait la question du devenir de ces enfants, et du devenir de la décision elle-même.

Dans une étude monocentrique, nous avions montré que les décisions prises en réanimation n'étaient pas pérennes et étaient rejugées à distance en tenant compte de l'évolution de la situation du malade et notamment de sa dépendance [12]. L'objectif de cette présente étude était de compléter cette analyse quantitative par une approche qualitative permettant de comprendre les mécanismes de l'oubli des décisions prises en réanimation, dans une perspective d'amélioration de la continuité des soins.

\section{Matériel et méthode}

Nous avons réalisé une analyse de contenu $[13,14]$ à partir du discours obtenu lors d'entretiens semi dirigés effectués auprès des médecins seniors qui avaient été en charge des enfants rapportés dans l'étude précédente [12], sortis vivants de réanimation après un questionnement sur d'éventuelles LAT. Des représentants de toutes les disciplines sollicitées dans cette étude l'ont été à nouveau. Huit mois ont séparé les deux études. 


\section{Entretiens}

Les entretiens se sont déroulés sur le lieu d'exercice des répondants. Les entretiens menés à l'aide d'un guide d'entretien construit à partir des thèmes mis en évidence lors de l'étude précédente [12]. Ils étaient enregistrés sur cassettes audio et intégralement transcrits. La durée était laissée à la convenance des répondants. Deux thèmes étaient systématiquement abordés : les décisions de LAT en réanimation pédiatrique et dans leur propre service. A la fin de chaque entretien les éléments non verbaux (interruptions volontaires ou non, attitudes des répondants, éléments de communication non verbale) faisaient l'objet de notes complémentaires.

\section{Méthode d'analyse}

Les discours des répondants ont ensuite été traités selon la méthodologie de l'analyse thématique $[13,14]$. Les thèmes communs, récurrents, à chaque entretien étaient relevés, indexés et classés.

\section{Présentation des résultats}

Les noms, date et lieux ont été rendu anonymes, et les éléments du discours trop personnalisés qui ne pouvaient être rendus anonymes ont été supprimés. Le discours des médecins est cité entre guillemets et en italique dans le texte. L'existence d'éléments supprimés au sein des citations est indiquée par le signe suivant : [...]. Les insertions dans les citations utiles à la compréhension du discours sont indiquées entre crochets selon le modèle suivant : [note d'auteur].

\section{Résultats}

Neuf médecins référents d'enfant sortis de réanimation après une décision de LAT ont été interrogés, neuropédiatres, neurochirurgiens pédiatriques, oncologues pédiatres, et hématologues pédiatres, aucun n'a refusé. Les entretiens ont duré en moyenne 45 minutes (35 -60). La transcription des entretiens constituait un corpus d'environ 80 pages. 


\section{A. Le médecin extérieur face au protocole de formalisation de la décision de LAT en réanimation}

\section{A. L'expertise}

Les médecins étaient tous intervenus en réanimation à titre d'expert, pour confirmer la gravité du pronostic et tenter de prédire l'évolution clinique avant une décision de LAT. Aucun médecin ne se définissait spontanément comme expert : ils disaient être venus en réanimation pour « donner un avis », avoir été consultés « pour donner les statistiques connues », ou avoir été « régulièrement impliqués dans la décision ». Lorsque le terme d'expert était suggéré, tous les médecins se reconnaissaient dans cette définition, probablement parce qu'elle faisait écho au champ sémantique des recommandations dont ils connaissaient les principes. La difficulté de se définir comme expert suggère les tensions qui existent autour de ce rôle.

\section{1/ Les tensions en objectivité et subjectivité}

L'expertise consiste à rendre un avis pronostique en confrontant les données cliniques aux données de la littérature. Elle répond à une nécessité d'objectivité de l'acte médical et s'appuie sur des données validées par la médecine basée sur les preuves. Quoiqu'il s'agisse d'un exercice habituel, les médecins interviewés rapportaient le malaise que générait l'acte d'expertise visant à établir un pronostic sur un sujet particulier :

«Quand on a des données sur l'ensemble d'une population, ce n'est pas parce que 50\% des enfants sont comme ça, que ça veut dire qu'à titre individuel un enfant sur deux sera comme ça. » [Neuropédiatre b] « Il est sûr qu'on généralise autour de statistiques, et ça, ça me met toujours mal à l'aise. » [Neurochirurgien a]

L'expertise - parce qu'elle a une dimension pronostique - vient ici se heurter à une incertitude intrinsèque. Or, c'est précisément à cet endroit d'incertitude que l'expertise est demandée en vue d'une décision. L'expertise devient alors un processus complexe qui doit intégrer les données scientifiques et annuler le mythe de la certitude :

«Si Toulouse-Lautrec était né en 2004, il ne serait jamais venu au monde, à la vue des malformations qu'on a pu décrire. C'est toute la complexité de tout ça : comment être sûr de ne pas être trop dans l'excès ?» [Neuropédiatre d]

«Le problème c'est que personne ne sait ce que les gamins vont devenir. [...]. Si les gens avaient 
l'honnêteté de dire, non pas je vais me tromper parce que je suis incompétent, mais bien parce que personne ne sait comment ça va évoluer...» [Neuropédiatre e]

Ces exemples suggèrent des limites dans l'évaluation pronostique de la maladie et dans la manière d'en rendre compte. Un autre point d'incertitude fragilise l'expertise : il s'agit de son interprétation par les réanimateurs et, donc, l'usage qui en sera fait. Ces deux niveaux d'incertitude confèrent à l'expertise un double niveau de subjectivité, qui est source de tension chez l'expert dénué de pouvoir de décision. Certains médecins dénonçaient l'hypocrisie du système :

«Moi je ne statue plus car cela ne sert à rien. En fait notre avis fait partie du protocole [...] et [à force] de voir à quel point c'est aléatoire, je me mouille de moins en moins. » [Neuropédiatre e]

«Parfois on a la sensation qu'on ne tient plus compte de notre avis. C'est variable en fonction du moment, du senior [de réanimation]....» [Onco-hématologue a]

« Je n'ai jamais eu d'information après sur comment c'était utilisé tout ça. [...] Je l'ai marqué. A eux de l'entendre ou de ne pas l'entendre !. » [Neuropédiatre c]

\section{2/ Le travail de distanciation et ses limites}

L'expertise demande un travail d'objectivation qui suppose une prise de distance par rapport au malade et son contexte, mais aussi par rapport à la décision qui pourrait être prise. Ce travail de distanciation était surtout décrit par les neuropédiatres, en référence au protocole de formalisation des décisions. Le protocole induit une séparation des actes d'expertise et de décision, qui pourrait permettre un certain équilibre dans le partage des responsabilités : celle de l'expert et celle du réanimateur à qui revient la décision :

« J'adhère au protocole tel qu'il est appliqué aujourd'hui, c'est-à-dire : on demande un avis expert, je donne

un avis expert que j'essaie [de rendre] indépendant. » [Neuropédiatre b]

«(...) je fais confiance aux réanimateurs, je considère que ce sont des médecins comme moi et donc [c'est] à eux de prendre des avis techniques et d'en faire la synthèse, pas à moi. » [Neuropédiatre c]

Mais dans la réalité, l'équilibre de la répartition des tâches (entre expertise objective et nécessité de « contextualiser » la décision) semblait assez précaire. Les neuropédiatres surtout rapportaient leur difficulté à trouver un équilibre entre l'objectivité de l'expertise, et la nécessité de connaître le contexte du malade. Leur proximité particulière avec le handicap, et donc avec la maladie chronique qui modifie progressivement les repères familiaux, les place dans une temporalité différente de celle des réanimateurs. Ils exprimaient le besoin d'une rencontre avec 
les parents mais étaient conscients qu'elle aurait pu faire « exister » la maladie chronique, alors que la décision de LAT n'était pas encore prise :

«[...] j'aimerais bien rencontrer les parents de ces enfants mais si je rencontre les parents, je perturbe la dynamique de soins de l'équipe de réanimation qui est fragile. ». [Neuropédiatre c].

« [...] Si on intervenait [en réanimation], on interviendrait sur [...] des gens qui se posent en permanence la question de vie ou de mort de leur enfant. [...] Je ne suis pas certain qu'on ait le degré d'expertise [pour cela]. » [Neuropédiatre b]

Le neurologue dans sa démarche d'expert se trouvait tiraillé entre la nécessité de «décontextualiser» au nom d'une objectivité scientifique et celle de tirer profit de son expérience de l'enfant handicapé et de sa famille :

«Deux situations se comprennent très bien sur le plan humain : [celle] du réanimateur, qui est là pour réanimer, et [celle] du neuropédiatre...C'est l'autre extrémité puisque c'est lui [le neuropédiatre] qui voit les séquelles de ce qu'a fait le réanimateur [...].

Pour moi, quand je vais en réanimation, j'ai deux situations : soit je connais l'enfant parce que c'était déjà un handicapé avant qu'il ait été réanimé, donc là [...], c'est l'environnement [qui compte] ; soit je ne le connais pas et, de fait, je vais faire intervenir des critères techniques identiques au réanimateur [...]. Je vais faire une démarche très technique [et je vais] dire : voilà le pronostic de cet enfant. Quand je vais en réanimation, j'ai une démarche de réanimateur parce que je ne connais pas le contexte, je suis comme eux [...]» [Neuropédiatre c].

Les onco-hématologues avaient moins de réticences à rencontrer les familles en réanimation, du moins au début du séjour. Leur intervention en qualité d'expert diffère de celle des neuropédiatres puisque la demande d'admission d'un de leur patient en réanimation intègre l'expertise oncologique et elle se justifie par l'existence d'un projet thérapeutique. Cependant lorsque l'aggravation de l'état de l'enfant entraînait la défaillance d'autres organes, le questionnement, et donc la demande d'expertise, se déplaçaient sur le devenir neurologique :

« Après [...], les gens sont contents de nous voir quand on va en réa parce qu'ils ont l'impression qu'il y a un suivi, qu'on les soutient malgré tout [...]. » [Onco-hématologue b]

«Q : et lorsque vous allez aux réunions décisionnelles, que pensez vous pouvoir apporter?

- déjà on a une connaissance de la famille au début de la réanimation, c'est sûr qu'on apporte quelque chose. Après, lorsque les enfants restent longtemps... » [onco-hématologue a]

\section{$B /$ Le protocole de formalisation : réticences et bénéfices ressentis}

Le protocole de formalisation des décisions de LAT semble permettre une répartition 
des tâches. Cependant la responsabilité de chacun n'en est pas pour autant diluée, et les médecins interviewés exprimaient à demi-mot une culpabilité conséquente :

« Je ne ressens pas de"degré de culpabilité" d'avoir fait une expertise tout seul dans mon coin, ou d'avoir participé à une discussion. Finalement, après, ils font ce qu'ils veulent de mon avis. » [Neuropédiatre b] Une des raisons de ce malaise était la solitude du médecin expert par rapport aux conséquences de son compte-rendu. En effet, si un collège de médecins se réunit pour porter ensemble une décision, l'expert, lui, « il la fait tout seul dans son coin l'expertise ». Les experts ne semblaient pas contester le fait d'être rarement invités aux réunions décisionnelles, jugeant que ce n'est pas leur rôle de «faire la synthèse »; on ne peut toutefois exclure que la raison en soit une certaine peur du conflit :

« Il y a eu une décision avec laquelle je n'étais pas d'accord. [...]. A cette occasion-là j'ai appris la culture pédiatrique, au départ on n'était pas d'accord mais on est resté sur leur décision. On n'était pas là pour se disputer. » [Neurochirurgien b]

La «protocolisation » de la décision étant fortement liée à la législation sur le sujet, beaucoup de médecins craignaient qu'il s'agisse d'une façon de légitimer une toutepuissance médicale, ou un droit de vie et de mort :

«Ce qui me gêne beaucoup c'est de se poser en juge souverain, le droit de vie ou de mort en quelque sorte [...]. J'ai toujours peur des dérives » [Neurochirurgien a]

« Je pense qu'il ne faudra jamais légiférer totalement sur ces problèmes profondément humains

[...], qui touchent un sujet différent d'un individu à l'autre. » [Neuropédiatre c]

«Je pense que ce qui est important, c'est de [...] ne pas être prisonniers de critères pré établis [...]. De façon assez générale dans les décisions d'éthique, en théorie, c'est très compliqué et en pratique la décision s'impose d'elle-même. Dès qu'on tente de s'abstraire pour faire quelque chose de théorique... [Silence] » [Neurochirurgien b]

De plus, la séparation des rôles d'expertise et de décision provoquait un malaise probablement lié à une assimilation implicite du protocole de formalisation avec un algorithme décisionnel. En réalité le protocole suggère des étapes de discussion mais ne peut en rien garantir la qualité de la décision. Tous les médecins avaient une conscience aiguë du risque de s'enfermer dans des critères fixes, pré-établis et de la nécessité de décider pour un malade particulier dans une histoire particulière. Certains reconnaissaient le protocole de formalisation comme un processus de discernement garantissant que toutes les conditions sont réunies pour prendre une décision : 
« Alors, même si on protocole les choses, on ne maîtrise pas tout. II n'y a pas de réponse dogmatique à avoir mais il faut rester au cas par cas... » [Neuropédiatre d]

«Léonetti [Député rapporteur de la loi], il a donné des garde-fous [...] Des garde-fous c'est parce que sinon le fou ne voit pas qu'il y a un précipice. Donc le garde-fou, étymologiquement, c'est pour garder le fou de tomber $[. .$.$] (Silence) » [Neuropédiatre c]$

L'objectivité qu'exige l'expertise est en permanence mise en difficulté par la subjectivité nécessaire à la connaissance plus globale du patient. Or les enfants sujets de décisions de LAT sont souvent pris en charge par plusieurs spécialistes, ce qui ne favorise pas l'approche globale :

« [...] la médecine actuelle qui parcellise le savoir, il y a le pneumologue, le cardiologue, le viscérologue... et que l'enfant dans sa complexité et son entièreté justifie un lien très fort entre le vécu, le psychisme et la maladie. » [Neuropédiatre c]

La sur-spécialisation médicale pose également le problème de la culture médicale qui influe notamment sur les représentations de la qualité de vie. Un onco-hématologue posait le problème en termes d'habituation :

« [...] c'est deux mondes complètement différents, les limitations de traitements pour nous et en réanimation. Pour les neuropédiatres c'est encore autre chose. Quand j'étais interne je suis passée en neuropédiatrie et j'avais du mal avec les encéphalopathies convulsivantes et quand il y avait un enfant avec tumeur cérébrale c'est les neuropédiatres qui déprimaient. » [onco-hématologue a]

Les différents médecins interviewés reconnaissaient que la concertation multidisciplinaire permet de faire converger les points de vue de chacun. Elle permet de minorer les erreurs d'appréciation de l'état de l'enfant et conforte les médecins dans l'idée « du mieux possible » pour le patient et de la « bonne » qualité de prise en charge du malade :

" On reste imprégné des pathologies qu'on soigne le plus souvent. [...], c'est l'intérêt de se rencontrer, [puisque le risque c'est] de faire des erreurs d'appréciations lorsque qu'on ne voit pas régulièrement les pathologies. » [Neurochirurgien a]

«(... ) En réa je ne me considère pas comme un expert mais comme un des soignants. Moi je crois que c'est plus qu'un expert.» [neurochirurgien b]

L'obligation faite par la loi d'inscrire et de motiver les décisions dans les dossiers était perçue comme un bénéfice garantissant une meilleure continuité des soins. II persistait cependant un malaise chez le médecin de garde n'ayant pas participé à la discussion qui se trouvait dans une position d'exécutant :

« [A propos de la nécessité des transmissions avec l'équipe de garde] Pour que ce ne soit pas une personne 
qui décide comme ça : on reprend l'alimentation...

L'enquêtrice : - vous souhaitez que les choses soient claires entre vous...?

- Oui, c'est ça en particulier la nuit. » [Neurochirurgien b]

«C'est difficile pour moi, j'ai mon avis et ce n'est pas mon service, donc je suis obligé de faire et ça je suis très mal à l'aise par rapport à ça » [Neuropédiatre a]

La formalisation semble être ressentie comme un facteur de qualité du processus de décision : chaque protagoniste est défini (médecin expert, médecin référent en réanimation), les éléments de discussion sont posés, et la réunion de concertation ouvre un espace de parole. Malgré la persistance de tensions liées à la difficulté de l'exercice d'expertise, la formalisation permet aussi à chacun de conscientiser les enjeux dans les cas extrêmes.

\section{B. Médecin expert, médecin soignant : négociations et pérennité de la décision}

i.Le médecin soignant transfert son malade en réanimation : qui est expert ?

Les neuropédiatres, mais surtout les onco-hématologues sont amenés à demander le transfert en réanimation pour leur patient. En général, celui-ci a déjà bénéficié d'une mobilisation médicale importante. Si l'indication du transfert en réanimation a été la plupart du temps discuté par l'ensemble de l'équipe qui le demande, celui-ci nécessite souvent une négociation avec le réanimateur. Bien que les malades d'onco-hématologie soient pris en charge dans la durée (et donc avec une certaine chronicité), le protocole thérapeutique en hématologie s'apparente davantage au modèle de prise en charge des maladies aiguës dont la séquence habituelle est: symptômes-diagnostic-traitement-guérison. Le transfert en réanimation est donc inscrit dans le «protocole » de traitement de l'enfant, et se justifie par le fait qu'il existe un espoir de guérison de la maladie onco-hématologique :

«Mais ce qui me gêne surtout lorsqu'on descend les enfants en réanimation c'est qu'on nous demande «quel est le pronostic de cet enfant?» parce que globalement si le pronostic est mauvais, on garde les enfants dans l'unité [d’onco-hématologie]. » [Onco-hématologue a]

«C'est complètement évident que si on transfère le malade en réa, c'est qu'on en a parlé entre nous et qu'on est persuadé [...] que c'est un cap aigu à passer. » [onco-hématologue b]

Le point commun entre les patients ayant un poly-handicap pris en charge en neurologie pédiatrique et ceux ayant une affection onco-hématologique est que les malades survivants 
retournent ensuite dans leur service respectif avec leur affection sous-jacente. Les médecins qui les adressent craignent que leurs patients soient stigmatisés en termes d'incurabilité par les réanimateurs. Cette crainte renforce la nécessité d'argumenter et de justifier le transfert en réanimation, ce qui accentue les difficultés déjà vécues par le médecin soignant face à la dégradation de l'état de son patient à moins que le service de réanimation devienne le lieu où se gère la crise :

"On ne va pas leur envoyer un malade alors qu'on sait qu'il va mourir dans les heures qui suivent. Ou alors c'est parce que on serait en grande difficulté avec la famille ; je n'en sais rien. » [Onco-hématologue b] «Ce qui me gêne beaucoup plus c'est la représentation qu'a le service [de neuropédiatrie] dans les autres services de pédiatrie en tant que service s'occupant d'handicapés donc d'enfants incurables. [...] le concept d'incurabilité par rapport au traitement agressif. [...] ça renvoie à la notion d'échec, de monstruosité [...]» [Neuropédiatre c]

Les neuropédiatres sont davantage confrontés aux maladies chroniques. Ils sont donc particulièrement habitués à n'avoir pas la guérison pour objectif. Contrairement aux malades d'onco-hématologie le transfert en réanimation repose sur des critères subjectifs (représentation de la vie de l'enfant par la famille etc...). De plus, les patients de réanimation survivants à une décision de LAT étant la plupart du temps pris en charge en neurologie pédiatrique, il existe une sorte d'équilibre des flux entre les deux services. Il semble que la formalisation des décisions en réanimation ait favorisé une conscientisation des problématiques de prise en charge de ces enfants : les réanimateurs comme les neuropédiatres ayant acquis une proximité plus grande avec la problématique propre à chacun des deux services :

«Avant on avait des refus de la part des réanimateurs, maintenant il n'y a plus de refus, il y a des discussions, c'est ça aussi qui évolue dans les choses. Avant, la discussion n'était pas tellement possible parce que de toute façon c'était un enfant neuro au départ. » [Neuropédiatre a]

\section{ii.A la sortie de réanimation : quand les experts deviennent des soignants}

\section{L'effacement du séjour en réanimation}

Le transfert de l'enfant du service de réanimation vers le service d'aval provoque une rupture liée au changement d'équipe et de projet de soin et concerne essentiellement les services de neurologie et de neurochirurgie pédiatriques. Dans le service d'aval, le médecin 
expert devenu soignant semble souvent le seul à connaître l'histoire du malade en réanimation. Quand les transmissions existent, celles-ci sont assez évasives :

"L'équipe paramédicale ne le connaît pas [le patient], et seuls les médecins concernés [le connaissent]» [Neuropédiatre d]

« Non, [les infirmières] ne savent pas forcément. Ni même mes collègues. [...]. Non, ce n'est pas un choix, c'est comme ça. L'enfant vient et on le prend en charge, c'est un peu ça quoi. Non, non, ils ne savent pas qu'il y a eu une limitation. » [Neuropédiatre b]

II semble qu'il y ait presque une volonté d'effacer le séjour en réanimation et les décisions prises. L'enfant ayant survécu incarne tant de souffrance qu'il serait humainement insupportable de remettre en question son existence après la tragédie de son parcours :

«On se les réapproprie mais le problème, c'est qu'il faut gérer l'équipe, parce que c'est extrêmement éprouvant pour toute l'équipe. » [Neurochirurgien b]

« Ce qui me ferait peur, [...], c'est qu'ils [l'équipe soignante] se disent : nous on va prendre en charge un enfant qu'on aurait peut-être pas dû prendre en charge parce qu'il a survécu [parce qu'il n'aurait pas dû survivre]. Est-ce qu'elles vont se démobiliser ? » [Neuropédiatre b]

L'effacement du séjour en réanimation peut aussi être le témoin d'un désir de protection du patient et de sa famille. Rester silencieux sur ce passé douloureux permet de respecter le cheminement des parents, et ainsi de leur donner la liberté de s'approprier l'histoire de leur enfant avec la pudeur et la prudence nécessaire à l'acceptation de la maladie :

« Non, ils n'en parlent plus. Ils ont appris à vivre le moment présent en réanimation et nous poursuivons cela et on suit l'évolution » [neurochirurgien a]

« (...) quand il y a eu ce questionnement en réa et que finalement l'enfant n'était pas si mal et qu'il a survécu, non, ce genre de choses ils ne l'ont pas oublié. Éventuellement, on aborde un peu le sujet quand on se rencontre, [quand] ils ont envie d'en parler, mais ça ne va pas plus loin. » [Neurochirurgien b]

Lorsque l'enfant ayant déjà un suivi médical lourd est sorti sans séquelle de réanimation, ce séjour semble être une parenthèse dans l'histoire de la maladie : il est vain de ressasser cette période inquiétante et plus aisé de reprendre le cours du traitement qui symbolise la possible guérison :

"[Le lien entre les familles et l'équipe soignante] se fait très bien car les parents ont l'impression de sortir... [Silence], d'être des enfants miraculés sortis d'une espèce d'enfer ; alors le lien se refait très vite. » [onco-hématologue a]

«C'est juste un cap aigu à passer et après, ça va bien se passer pour lui et il va être dans les $70 \%$ qui guérissent et voilà. » [Onco-hématologue b] 
En neurologie, «l'enfant d'avant » n'est la plupart du temps pas connu. L'enfant survivant est un autre enfant dans le sens où le handicap et/ou la maladie chronique bouleverse les repères familiaux et implique un nouveau projet de soin :

«Ce qui s'est passé est passé et l'organisation des soins après est différente mais ça n'empêche pas le confort, le sens de l'existence de l'enfant, de ses parents, etc.... c'est un autre cadre. » [Neuropédiatre a]

«On les prend tels qu'ils sont. » [Neurochirurgien a]

«II ne faut pas avoir de regrets, quoi qu’on ait décidé, quoi qu'il se soit passé. Après, les choses s'imposent d'elles-mêmes, la vie reprend son cours.» [Neurochirurgien b]

Quelle que soit l'histoire médicale de l'enfant, les discours convergeaient : on parle peu du séjour en réanimation. Mais le nouveau projet de soin reste fragilisé par cette douloureuse étape.

\section{L'équilibre fragile autour du nouveau projet de soin}

La survie de l'enfant pour lequel une décision de LAT a été prise génère des difficultés et semble remettre en cause la décision prise. Quelquefois, la survie est illogique : l'enfant « aurait dû mourir ». A l'opposé, la survie de l'enfant peut signifier l'erreur d'appréciation de son état au moment de la prise de décision : «on s'est trompé, on n'aurait pas dû imaginer qu'il meure ». Le projet de soin est d'autant plus clair et réapproprié par les services d'aval que cela a été discuté au moment de la sortie avec les réanimateurs :

« A partir du moment où ils sont arrivés chez nous, a priori, ça remet en cause le questionnement initial. Mais il y a eu des cas où aussi on s'est rendu compte que c'était un peu forcé et on s'est dit, on ne retourne pas en réa, on met les choses au clair avec les parents» [neurochirurgien b]

«La seule chose qui est éventuellement transmise, c'est que cet enfant ne reviendra plus en réa. Ça a été discuté et décidé. » [Neuropédiatre b]

La sortie de réanimation d'un enfant ayant survécu alors qu'une décision de LAT avait été prise signe l'entrée dans la maladie chronique. Après les bouleversements liés au séjour en réanimation, la famille va progressivement se réapproprier l'enfant avec la maladie chronique qui bouscule les repères familiaux et sociaux. La famille devient un des acteurs de santé et mène un travail d'ajustement de son projet de vie. Ce travail lent et progressif, fragile aussi, nécessite une mobilisation positive de la famille et des soignants qui l'entoure :

«On ne va pas leur asséner tous les jours qu'il va y avoir des séquelles. On fait le point avec eux et on se réjouit de ce qui va bien. [...] » [Neurochirurgien a] 
«Quand [les parents] arrivent ici, il faut reconstruire, qu'ils soient prêts ou pas. [...]. Mais il y a quand même des choses qui ont été laissées derrière, même s'ils n'ont pas encore mis le pied dans l'avenir. » [Neuropédiatre b]

« [À propos du transfert en neuropédiatrie après la réanimation] Parce que c'est une autre étape, les enfants sont là, les parents sont là, et il faut les aider aux mieux (...). » [Neuropédiatre a] 


\section{Discussion}

Cette étude qualitative décrit pour la première fois la perception par les médecins des autres spécialités des recommandations du GFRUP concernant les LAT en réanimation pédiatrique [8]. Elle a permis de caractériser les tensions pressenties lors de notre précédente étude [12], et d'explorer ainsi les limites de ces recommandations auprès des collègues des autres services. Trois situations génératrices de tensions ont été identifiées, correspondant aux trois situations au cours desquelles les médecins interrogés ont été directement confrontés au protocole de formalisation de la décision de LAT en réanimation pédiatrique. La situation la plus fréquemment rapportée était leur intervention à titre d'expert à la demande des réanimateurs, pour confirmer la gravité du pronostic et tenter de prédire l'évolution clinique avant la prise de décision de LAT. Des tensions ont également été rapportées à l'admission en réanimation lorsque ces médecins étaient amenés à justifier un projet de soins, et après la sortie de réanimation lorsque le patient survivait après une décision de LAT.

Les tensions en rapport avec la demande d'expertise pronostique ont été les plus fréquemment citées. Dans le service de réanimation pédiatrique étudié, cette expertise était systématiquement demandée avant toute réunion décisionnelle [11], en conformité avec les recommandations du GFRUP [8]. En revanche, l'expert n'était généralement pas invité à la réunion décisionnelle, ce qui n'est d'ailleurs pas formellement recommandé par ce texte [8]. La modélisation décisionnelle comprenait donc une séparation volontaire, intellectuelle et physique, entre l'expertise scientifique et l'acte de soins. Dans cette situation, les deux pôles de l'activité médicale que sont l'objectivation (définie par Mallet comme la transcription de la plainte du malade dans un référentiel scientifique préexistant [15]) et l'attention à la subjectivité du malade et de sa famille (définie par le même auteur comme le fait de considérer la maladie en tant qu'événement biographique et social) étaient artificiellement séparés dans le temps et dans l'espace. Ils étaient incarnés respectivement par l'expert d'une part et l'équipe de réanimation d'autre part. Dans ce contexte, il n'est pas surprenant que les neuropédiatres, qui ont en charge des enfants polyhandicapés pour lesquels ils cherchent à « pondérer l'espérance de vie par la qualité de vie » [16] soient les plus enclins à déplorer l'absence de contact avec les 
familles lors de leurs interventions en réanimation. Cette répartition des rôles était d'autant plus génératrice de tensions chez les médecins appelés en tant qu'experts qu'ils n'ignoraient rien des buts de l'expertise et qu'ils connaissaient a posteriori l'usage qui en avait été fait. Leur expertise était ainsi biaisée puisque nuancée par l'utilisation qui risquait d'en être faite, avec un mode d'adaptation consistant parfois à ne pas trancher pour ne pas être instrumentalisé.

Il est important de noter que le législateur n'a pas repris à son compte cette séparation entre l'expertise et la décision dans le décret d'application de la loi de 2005 [10] qui définit la collégialité minimale pour les décisions de LAT. En effet, dans les articles R.4127 à R.4137 du code de santé publique intégralement repris dans le nouvel article 37 du code de déontologie médicale il est indiqué que : «La décision est prise par le médecin en charge du patient, [...] sur l'avis motivé d'au moins un médecin, appelé en qualité de consultant. ». Dans son commentaire de l'article 37 du code de déontologie le conseil de l'ordre des médecins précise que le consultant est un médecin « qui n'est pas obligatoirement un spécialiste ou un expert de la question, mais qui, par sa présence auprès du malade, conforte les proches dans l'assurance qui leur est due que la décision sera, en tout état de cause, prise de manière raisonnée et raisonnable » [17]. Cette formulation, qui suppose une rencontre avec le patient (et donc, en pédiatrie, avec ses parents), ne laisse guère de doute quant au fait que l'intervenant extérieur doit s'impliquer dans la décision. La participation du médecin expert à la réunion décisionnelle lui donnerait une vraie dimension de consultant au sens du code de déontologie.

Les recommandations du GFRUP soulignent que l'examen de la situation médicale du malade doit toujours constituer le premier temps de la réunion décisionnelle [8], mais elles ne posent aucune exigence de certitude absolue quant au pronostic. Cette priorité donnée à l'analyse des facteurs médicaux vise à permettre de repérer les faux problèmes éthiques qui pourraient trouver leur solution sans sortir de la « logique médicale » [8], et à faire valider par tous les participants le caractère extrême de la situation [18]. Elle vise également à protéger les participants non médecins d'un dépassement de leur rôle propre [19], puisque ce n'est qu'après que les médecins ont constaté l'impossibilité de faire le bien du malade en ne considérant que des facteurs médicaux, qu'ils sont invités à participer à la discussion prenant en compte d'autres arguments. La présence de l'expert à la réunion décisionnelle pourrait probablement 
résoudre une partie des tensions générées par l'incertitude sur le pronostic qu'on leur demande d'établir. Cette incertitude serait plus facilement intégrée dans la démarche décisionnelle à l'occasion d'une discussion argumentative entre tous les acteurs de soins et l'expert, que par la rédaction d'un compte-rendu clinique inscrit dans le dossier du malade.

La deuxième situation génératrice de tension concernait l'admission en réanimation des enfants atteints de maladie chronique. Le discours des médecins interrogés (en particulier celui des onco-hématogues) montrait que les demandes de précisions pronostiques des réanimateurs pouvaient être perçues comme une remise en cause du projet de soins.

La notion de plan de traitement est une particularité notable de l'onco-hématologie dont les spécialistes utilisent une métaphore militaire en parlant de lignes de traitements successives, comme des lignes de défense opposées successivement à un envahisseur. L'escalade thérapeutique est planifiée dés l'induction de la première chimiothérapie et les modifications stratégiques prévues en fonction de la réponse de la maladie. L'admission en réanimation pédiatrique est généralement demandée lors de la survenue d'une complication du traitement, ne modifiant le plan de traitement cancérologique initial qu'en le retardant. Ainsi, peut on lire dans un article publié par des onco-hématologues pédiatriques américains : " pour la majorité des enfants atteints de cancer, le but des médecins est d'arriver jusqu'au terme du traitement et que les considérations quand à la toxicité de la thérapeutique, la qualité de vie, la croissance et le développement sont habituellement mises au second plan » et qu'il est « difficile pour les médecins ayant en charge ces enfants de changer leur point de vue tant qu'il persiste un petit espoir de traitement curateur » $[11,12,28]$.

Dans ce contexte, il n'y aurait donc pas lieu de discuter l'admission d'un malade en réanimation s'il reste des lignes de traitements à proposer. Les choses ne sont pas aussi tranchées, comme en témoigne le fait que la Société Française d'Hématologie a publié en 2005 des réflexions sur la limitation des traitements en hématologie [21] qu'elle présente comme inspirées des recommandations de la Société de Réanimation de Langue Française [22].

La rationalisation de la démarche, prenant en compte tous les arguments médicaux et scientifiques est incontournable pour améliorer la qualité des décisions. Le premier axe de cette 
démarche vise à réduire l'incertitude pronostique. L'amélioration des scores pronostiques adaptés aux patients d'onco-hématologie serait très utile et pourrait servir de critère pertinent d'adaptation des stratégies thérapeutiques pour cette cohorte de malades [23]. Le deuxième axe de cette démarche a pour objectif de mettre à jour en permanence la connaissance de l'efficacité des stratégies thérapeutiques. Ainsi, un article récent a démontré l'amélioration globale de la survie des enfants immunodéprimés bénéficiant d'une ventilation mécanique en réanimation au cours des dernières années, mais a confirmé le caractère péjoratif de la coexistence de plusieurs défaillances d'organe [24]. Cet article a fait l'objet d'un éditorial qui en soulignait l'intérêt pour améliorer les critères de transfert en réanimation de ces malades, mais aussi les décisions de LAT [25].

Cette approche rationnelle n'est pas incompatible avec une approche multidisciplinaire, tenant compte de la subjectivité et de la temporalité de chacun des acteurs. L'admission d'un malade d'onco-hématologie en réanimation est une situation presque caricaturale où les deux pôles de l'exercice médical que sont l'objectivation et l'attention à la subjectivité [26] se trouvent brutalement confrontés, incarnés par des équipes soignantes différentes. Dans cette situation les onco-hématologues proposent aux réanimateurs un patient qui n'a pas encore parcouru le chemin que la médecine avait prévu pour lui et qui est victime d'un incident de parcours. En miroir, les réanimateurs accueillent un patient avec un parcours jugé lourd, pour qui il leur est demandé de mettre en œuvre des techniques agressives et incertaines. Dans cette situation, les réanimateurs habituellement considérés comme des techniciens se trouvent de fait les défenseurs d'une certaine subjectivité. Si la délibération des soignants autour du malade qui rendue nécessaire par cette confrontation n'est pas esquivée, ou travestie en discussion exclusivement pronostique, on peut en attendre un rééquilibrage bénéfique pour le malade et une meilleure connaissance du mode de fonctionnement de chaque équipe que les oncohématologues appellent de leurs vœux [11,12, 28].

La troisième situation génératrice de tensions correspond à la prise en charge des enfants à la sortie de la réanimation. La possibilité de survie après une décision de LAT en réanimation est une donnée récente. Par définition cette possibilité n'apparaissaient pas dans 
les études sur les modes de décès en réanimation $[11,12,28]$; elle n'a été mise en évidence qu'à la suite des études prospectives récentes ayant inclues les malades au moment du processus de décision $[11,12,28]$. Ainsi, dans une étude prospective réalisée dans 113 services de réanimation d'adultes français, et concernant 807 adultes pour lesquels il y avait eu décision de LAT, 22\% d'entre eux survivaient [28]. De même dans une étude prospective de deux ans réalisée dans le service de réanimation pédiatrique concerné par la présente étude, 11 des 31 enfants ayant fait l'objet d'une décision de LAT étaient sortis vivants de réanimation [11]. Dans une autre étude s'intéressant au devenir des malades à la sortie de réanimation, nous avions confirmé que 30\% des malades ayant fait l'objet d'une décision de LAT étaient sortis vivants de réanimation. Leur niveau de dépendance estimée par le score Pediatric Overall Performance Category (POPC) [29], correspondait à une dépendance totale à l'entourage pour les actes de la vie de tous les jours [12]. La présente étude confirme que les équipes en charge des enfants après leur sortie de réanimation ne prennent pas en compte le questionnement antérieur au sujet d'éventuelles LAT. Elle montre qu'il ne s'agit pas d'un simple dysfonctionnement technique en mettant à jour les mécanismes qui président à cette rupture dans la prise en charge du malade.

Le transfert en neurologie pédiatrique de patients avec de lourdes séquelles neurologiques, a fortiori s'il y a eu questionnement sur d'éventuelles LAT, réalise une deuxième situation de confrontation, où les réanimateurs jouent un rôle inversé par rapport à la situation de l'accueil d'un malade d'hématologie décrite plus haut. Dans cette situation, le souci de la subjectivité est incarné par l'équipe de neurologie qui a la charge de reconstruire un projet en tenant compte de la capacité de la famille et de la société à réinvestir le patient, alors que la nécessité d'objectivation est incarnée par l'équipe de réanimation qui s'est questionnée sur l'utilité de la prise en charge. Là aussi, les tensions générées par cette confrontation, pourraient déboucher sur une délibération profitable au patient. La participation du médecin expert aux décisions de LAT en réanimation pourrait favoriser cet échange. La présentation de l'enfant à l'équipe du service de neuropédiatrie par un réanimateur qui s'y déplacerait, tel que cela a été suggéré lors des entretiens, irait dans ce sens, et pourrait transformer les négociations entre services en délibérations autour du patient. 
La généralisation du recours au consultant que devrait induire la loi [10] sera un facteur favorisant la transdisciplinarité des décisions, s'il est choisi à bon escient, soit au sein des médecins qui ont adressé l'enfant en réanimation, soit au sein de l'équipe qui pourrait en être le destinataire en cas de survie. De même rien ne s'oppose à ce qu'un réanimateur joue le rôle de consultant en amont, lors d'une décision de ne pas transférer une enfant en réanimation ou de le transférer avec des objectifs limités et déterminés à l'avance, et régulièrement évalués par les deux équipes.

Cette étude comporte plusieurs limites. Elle a été réalisée par une interne de pédiatrie en fonction sur le terrain de l'enquête et ayant été en stage dans la plupart des services impliqués, ce qui a pu entraver sa neutralité. En revanche, cette particularité de statut lui a permis de satisfaire à deux critères de qualité d'une enquête de terrain : l'immersion dans le milieu et l'imprégnation [30]. Le corpus était limité et la saturation des thèmes n'a probablement pas été atteinte. Les entretiens se sont limités à des médecins seniors, appartenant aux services impliqués dans la précédente étude [12] ; les propositions pratiques qui en découlent ne sont donc probablement pas directement transposables dans une autre structure que celle de l'étude. Cette étude doit être considérée comme préliminaire à une étude multicentrique dont le financement vient d'être assuré par l'obtention d'un PHRC en 2008.

\section{Conclusion}

L'hospitalisation en réanimation est un moment de crise pour le patient et sa famille, mais aussi pour les équipes soignantes qui l'adressent en réanimation ou qui le prennent en charge à sa sortie, a fortiori s'il y a eu une réflexion concernant une éventuelle LAT. Les recommandations du GFRUP proposent des éléments d'éthique procédurale pour favoriser la prise de décisions dans les situations extrêmes de la réanimation. La modélisation du raisonnement médical qu'elles utilisent semble adaptée à la réanimation, mais elle est source de tensions quand elle est confrontée aux modélisations des autres spécialités. Ces modélisations représentent des modes d'intégration différents de la subjectivité présente dans 
tout raisonnement médical. II serait illusoire de prétendre éliminer cette subjectivité. En revanche, sa réintégration dans la pratique, éventuellement même réduite à une meilleure connaissance des modélisations des autres spécialités, serait de nature à favoriser la transdisciplinarité et le partage d'informations décisionnelles nécessaires à la continuité de sa prise en charge. 


\section{Références}

http://www.conseil-national.medecin.fr 